\title{
Research and development in quality of care: establishing the research agenda
}

\author{
Richard Grol
}

\begin{abstract}
Introduction
Quality of care has, in the past decades, evolved into a new important field of work including activities such as quality assurance, clinical audit, quality improvement, and quality management. It has gradually become a domain with its own concrete aims, its own typical methods and techniques, and its own specific educational programmes, journals, societies, meetings, conferences, laws, and budgets. ${ }^{1}$ Russel and Wilson speak of clinical audit as "the third clinical science". ${ }^{2}$ Whether this is true or not, quality improvement and clinical audit pose at least new challenges for every person and institute in health care. New expertise is required: people who can provide education and support on quality improvement, such as audit facilitators and quality managers. New feasible and effective methods for quality improvement are also needed, such as methods for setting guidelines, reviewing or auditing performance, inducing changes in provision of care, and managing quality improvement actions. Consequently a new field of research and development has evolved, aimed at optimising the quality of patient care. In some countries even specific research budgets for research on quality of care and quality improvement have been established (The Netherlands, Norway, United Kingdom). From this, questions arise about the specific role of research on quality of care and quality improvement. Is it different from clinical research or health services research; and what are its specific features and research questions?

This paper, for researchers and policy makers, provides a reflection on the role of research and development in quality of care, which is characterised by its own particular subject and research questions.
\end{abstract}

Features of research into quality of care Research and development in quality of care and quality improvement are closely linked to clinical research and research on health services, and so should not be characterised by specific research methodology, but primarily by research questions. These are derived from continuously assuring and improving the quality of patients' care. The aim of all parties in health care is that patients receive good or optimal care. Their views on good clinical practice may, however, differ. Professionals will emphasise the clinical effectiveness of their actions and good health outcomes; patients wish a friendly attitude in care providers, good information on their illness, and easy access to health services; whereas health authorities will stress efficiency and cost effective care. So, good quality of care includes a variety of aspects.

To deliver and ensure such care, specific measures are necessary to systematically and continuously evaluate and enhance the quality of care. This I shall call quality improvement. Quality improvement includes a variety of mutually related activities, such as tracing problems, developing guidelines, criteria, protocols, or targets for good clinical practice, reviewing actual performance, changing care when necessary, and creating the conditions for improving clinical practice.

Good clinical practice is a dynamic concept, which is continuously fed and supported by research and development (fig 1). On the one hand this refers to research on health and illness (study of the aetiology, incidence, prevalence, course, diagnosis, prevention, and treatment of diseases and symptoms). Input particularly comes through studies of clinical research and health technology which provide evidence on valuable procedures for diagnosis, treatment, and prevention. These may not only include the efficacy of the procedures, but also their safety, costs, ethical aspects, or social implications. Clinical practice is influenced on the other hand by research on health services. This is defined as: "a multidisciplinary field of inquiry, both basic and applied, that examines the use, costs, quality, accessibility, delivery, organisation, financing and outcomes of health care services and effects of health services for individuals and populations". So, this includes research on the actual quality of care and factors influencing the quality of care positively or negatively.

Both research on good clinical practice and research on the quality of health services provide a major input for research and development in quality improvement (fig 1). Research on the quality of healthcare attempts to provide scientific support for actual activities that improve quality. It studies which methods, tools, approaches, instruments, and programmes are most cost effective, feasible, and acceptable for assessing and improving the quality of patient care. Clinical research or studies on health technology may show the effects and costs of a new inhaler on lung function and the quality of life of patients with asthma. Research on health services may study the actual use of this medication and variation in prescribing between practitioners. Research on quality of care and quality improvement may focus on translating the evidence from clinical research into a feasible guideline, or into quality indicators, or devising a method to collect reliable data on the quality of asthma 


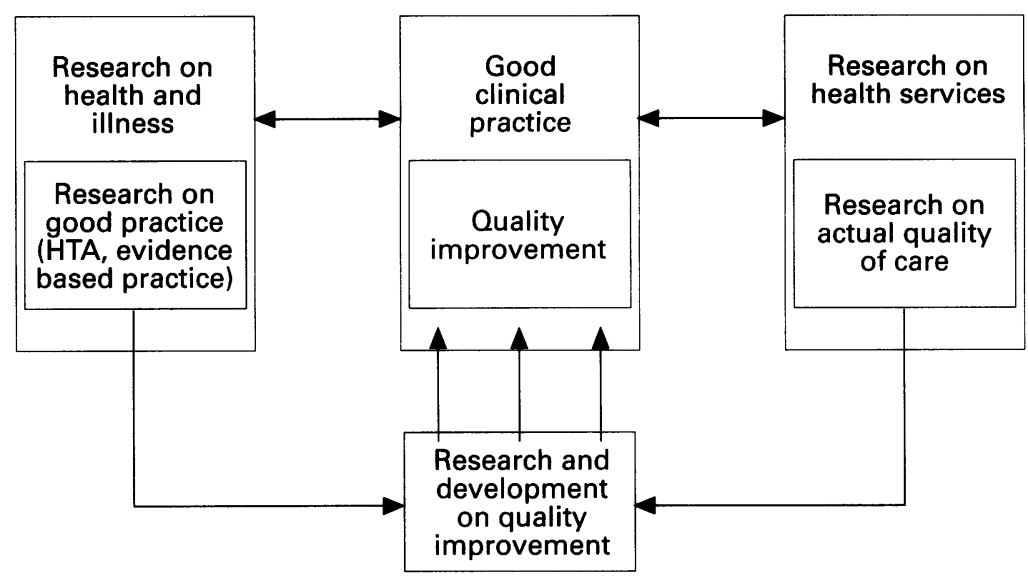

Figure 1 The place of research and development in quality improvement in the research field; HTA = health technology assessment.

medication. Alternatively it may focus on factors determining the variation in adherence to the new guideline on asthma medication, or compare the effects of an outreach visit by an experienced person with systematically reminding and feeding back on adequate prescribing.

Specific features of such research and development are that they are primarily concerned with assessing or changing actual care and implementing new routines. This implies that not only the efficacy or reliability of the tools are important, but also their feasibility for daily practice. Research might be on a method of visiting a nursing home and assessing performance in a reliable manner; on an instrument to assess patients' satisfaction to be used in audits in primary care; a feasible complaints procedure for hospitals; the costs and effects of a method to influence behaviour of physicians in ordering tests; the sensitivity of indicators to assess the quality of care of dentistry; the effects and feasibility of a method for achieving consensus between general practitioners (GPs) and hospital specialists on indications for referral; the development of an evidence based and feasible protocol for optimising cardiovascular prevention; the effects of a programme to implement improvements in home care organisations; the value of a recertification system, or the effects of specific formal policies and regulations to improve patient care.

Research on quality of care and quality improvement aims to find the implications of good clinical practice, which factors influence that quality of care, and how professional practice and institutional performance can best be improved to achieve such a high quality of care. It takes care of the translation of research on good clinical practice and uses results from research into health services to find possible problems in the quality of care and select methods to improve care effectively. ${ }^{4}$

Research on quality of care: which topics should be considered?

Research and development in quality of care is a relatively new research area, although considerable research efforts have been undertaken in the United States, the United Kingdom, and some other countries - for example, The
Netherlands and Scandinavian countries. Most of these studies concerned actual care and determining variations in provision of care.

Which topics need to be considered in research on quality of care? This depends particularly on the framework used to describe quality improvement. The framework used here is derived from a statement on systematic quality improvement developed for the Council of Europe (Expert Committee on Quality Assurance, unpublished report) It describes quality improvement from different angles:

- The actors in quality improvement

- The methods and procedures used in quality improvement

- The management of quality improvement: systems and conditions

These perspectives are not independent, but all initiate specific research questions.

(a)Actors in quality improvement:

What are the different views on good clinical practice of professionals, patients, authorities, etc?

How to involve patients, authorities, payers in setting guidelines and assessing the quality of care?

(b) Methods and procedures used in quality improvement:

- Identification of quality problems:

Validity, reliability, sensitivity, specificity of quality indicators

Value of data collection sytems aimed at tracing quality problems

Which factors determine good or bad quality? Value of methods to analyse possible quality problems (such as paretograms, cause-effect diagrams, etc)

- Development of clinical guidelines and criteria: (Cost) effectiveness of methods or procedures for guidelines development

Advantages or disadvantages of central $v$ decentralised guidelines

Advantages or disadvantages of mono $v$ multidisciplinary guidelines

Which features of guidelines determine acceptance and adherence?

- Data collection and evaluation of care: What sort of information on quality of care is needed by different parties in health care? Psychometric value of instruments and procedures for quality assessment Advantages or disadvantages of internal versus external assessment

Value of systems for licensing and accreditation

- Improving and changing practice:

Cost and effectiveness of methods and programmes for inducing change in clinical practice and implementation of guidelines Value of organisational development for quality improvement

Which factors determine the implementation of changes (the black box)?

(c) Management of quality improvement:

Which resources and support structures are required to implement quality improvement systems?

(Cost) effectiveness of courses to teach care providers methods of quality improvement Which organisational development is needed for setting up quality improvement systems?

Box 1 Examples of research issues and questions in research on quality of care 
- Opinions of both patients and GPs on high quality general practice care were gathered through surveys among a sample of $\mathbf{4 5 5}$ patients from 14 practices (response 55\%) and a random sample of 263 Dutch GPs (response 67\%)

- The respondents rated 40 different aspects of general practice care on a scale, running from not important at all to most important

- Comparison of the answers showed that the opinions of GPs and patients were partly similar - for example, providing quick services in case of emergencies - and were partly different Patients gave priority to having enough time during consultations, having an appointment within a short time, and getting sufficient information on their illness

- GPs gave priority to exploring and understanding patients' expectations well, a good cooperation between GP and the support staff, and GPs working according to accepted knowledge and evidence on good practice

Box 2 Priorities of patients and GPs on care in general practice (fung 1996, unpublished report)

\section{ACTORS IN QUALITY IMPROVEMENT}

Different groups of people play parts in quality improvement in health care and they may have different views on good clinical practice. Research should, for instance, clarify the different perspectives of professionals, patients, managers, payers, and health authorities on quality of care and study how these can complement each other. Research on quality of care should also explore the possible contribution of the various parties to setting guidelines, selecting quality indicators, assessing care, and changing practice.

\section{METHODS AND PROCEDURES USED IN QUALITY IMPROVEMENT}

Quality improvement uses specific procedures and processes, usually in a cyclical manner; these are basically four mutually related activities: identification of quality problems and selecting topics for improvement; setting guidelines and criteria; collection of data and evaluation of actual care; and implementing changes and improvement.

\section{Selection of topics, identification of quality problems}

Health care is a broad and diverse field; time for quality improvement is restricted; the number of topics endless. So, quality improvement should be focused at the most relevant aspects of care and at problem areas with the greatest impact on patients and care providers and the maximum chance of improvement. Research on quality of care should support the identification of these areas. So, research should be concerned with crucial indicators to evaluate the quality of care, particularly finding indicators for the outcome of care. Research on the validity, sensitivity, specificity, and acceptance of these indicators is required to find which are most relevant, which best predict differences in quality of care, and which most reliably measure quality of care?

Another type of research that evaluates methods for the collection of routine data may be particularly important to screen for problems in the quality of patient care or in
- To determine which aspects of general practice care can and should be evaluated by chronically ill patients, interviews were organised of small focus groups of chronically ill patients and GPs

- Agreement and disagreement between both groups were identified

- On the basis of these interviews a written consensus procedure was used to reach a better understanding of the various opinions

- A list of aspects of care in general practice for chronically ill patients was made and patients and GPs were asked to determine which aspects can be evaluated well by patients

- Aspects that reached $50 \%$ consensus were selected as quality indicators and were further developed

- Examples of indicators are:

Giving information on disease and treatment

Providing support in the acceptance of chronic illness

Taking care of an active surveillance of the disease

Collaboration between GP and specialist in management of the disease

- Different preliminary tests showed that:

Handing out questionnaires in the practices had a higher response than a postal survey $(72 \% v$ $63 \%)$

Acute patients were more critical than chronically ill patients on some aspects of general practice care

Box 3 Development of indicators for evaluation of general practice care by chronically ill patients ${ }^{5}$

areas where there is cause for concern. These may be methods for recording mortality and morbidity, gathering data on different practice activities, hospital registration, patients' complaints, or registration of mistakes and critical incidents. ${ }^{6}$ These data are now collected and analysed on a large scale. They are often used in healthcare policies, but their value for tracing quality problems and quality improvement processes at a practice or institutional level is not yet clear. Such data may also be used to identify specific factors that determine the quality of care; factors related to the care providers, to the work setting, or to the patients.

A last field of research related to tracing quality problems, evaluates new methods of identifying and defining problems derived from total quality management or continous quality improvement, such as control charts, paretograms, or cause-effect diagrams. Research evidence on these tools is largely lacking: but are they really helpful and effective in quality improvement processes in health care?

\section{Clinical guidelines and criteria for adequate performance}

After identifying possible problems in the quality of patient care, defining what is seen as desirable care is usually a first step in assessing actual care and the need for improvement. Different procedures are used in different countries, such as development of evidence based guidelines, national consensus conferences, expert groups using standardised procedures and systematic literature reviews (meta-analysis), local group methods, or Delphi procedures. Certain groups claim that 
- To find the reliability and validity of clinical guidelines developed in a written Delphi procedure for GPs to diagnose abdominal complaints, two mixed groups, each with $10 \mathrm{GPs}$ and specialists, independently developed consensus criteria for the management of abdominal problems through a structured Delphi procedure; coefficients for the degree of consensus between the two groups ranged from 0.84 to 0.96

- These guidelines were compared with the national guidelines for the management of abdominal complaints in general practice, developed by the Dutch College of General Practitioners through a rigorous, standardised procedure in which the scientific evidence is reviewed and a broad acceptance among GPs is achieved

- Generally, the results of the two procedures were similar, although the Delphi procedure resulted in more global guidelines

Box 4 Comparison of two methods to develop guidelines for clinical practice

evidence based or explicit approaches are better than national or decentralised consensus methods in developing clinical guidelines. Comparative research on the effectiveness of the different approaches is, however, lacking.

Research on clinical guidelines is usually restricted to the evaluation of a specific guideline: its use, feasibility, and effectiveness under normal care conditions. Research on methods to develop valid and feasible clinical guidelines is scarce. ${ }^{8}$ Research is required on methods to analyse the scientific literature effectively, on the cost effectiveness of methods to achieve consensus among involved parties, on advantages and disadvantages of setting single versus multidisciplinary guidelines, of setting centralised versus decentralised guidelines, and of involving non-professional people (patients, authorities) in the setting of guidelines. Developing instruments for a critical appraisal of guidelines is now under way. ${ }^{9}$

Particularly interesting are the costs involved in the different ways of setting guidelines. When comparing the costs needed for the development of a clinical guideline - for example, by the United States Agency for Health Care Policy and Research (cost per guideline ranged from $\$ 340000$ to $\$ 675000$ ) and by the Dutch College of General Practitioners (cost per guideline was $\$ 100000$ ) - the question is raised whether the difference in investment results in improved guidelines or not. ${ }^{10}$

Guidelines and standards are not only related to clinical performance, but may also be concerned with the organisation of services. For instance, what is the value and feasibility of International Standards Organisation (ISO) standards for organising services for hospitals and office based practices? Finally, a real challenge is the translation of usually globally defined clinical guidelines into valid, reliable, and feasible (medical) review criteria to be used in quality assessment. ${ }^{8}$ Such criteria are needed to evaluate whether the guidelines are actually followed and adhered to in daily care, and whether the guidelines lead to the expected health outcomes.
Experiences with developing two evidence based guidelines (management of recurrent wheeze in adults and management of stable angina) were outlined in a process analysis

- Two multidisciplinary teams (11 people) with patient representatives included, developed and reviewed the guidelines in about 18 months; each guideline cost about $£ 27000$

- Team members adopted different roles (group leader, specialist resource, technical support)

- A structured procedure was followed: Defining the scope of the guidelines

Review of the scientific evidence (Medline search 1985-94)

Deriving recommendations on the basis of grading the evidence

Consensus discussions

External review by experts and users (9 people) Final version

- It was concluded that development of a guideline requires specific skills and should preferably be performed at a national level, whereas at a local level the guideline can be appraised and adapted

- Patients should not be part of the working group, but should be involved in different roles

Box 5 Developing valid guidelines: methodological and procedural issues ${ }^{11}$

\section{Data collection and evaluation of actual care}

A next crucial step is the gathering of information on actual provision of care and evaluating care in relation to the guidelines and criteria. As in each measurement procedure, reliable and valid methods are needed. Practice visit methods, quality of life scales, patients' satisfaction survey methods, chart audit methods, and self recording instruments or skills tests used in evaluation of quality of care should meet specific psychometric criteria.

- To establish the validity of two questionnaires of patients' satisfaction of general practice (one on the surgery and one on the consultation) a prospective study was performed in 400 patients who had changed practices and 869 randomly selected patients who had not changed practices

- It was assumed that dissatisfied patients would be more likely than satisfied patients to change practice

- The results of the completed questionnaires in the two groups of patients supported this assumption

- For both questionnaires the patients who did not change practices had significantly higher satisfaction scores

Box 6 Measuring patient satisfaction: a test of validity ${ }^{12}$

Research on health and health care has given rise to many methods of measurement; these are usually only partly suitable for systematic use in quality assessment and improvement, because they have not been developed for that purpose. Procedures for use in quality improvement must not only be valid and reliable, but must be easily applicable in normal care settings and be acceptable to the care providers and institutions in question. Ideally, they will not only assess care, but also contribute to improvement which they must be sensitive enough to identify correctly. Research on new and old methods and their use in quality improvement is therefore a priority.

The research should aim at developing feasible methods of assessment, and also identifying factors that explain variations in performance 
- To assess the responsiveness to changes in health status over time for four clinical conditions (low back pain, menorrhagia, suspected peptic ulcer, and varicose veins) postal questionnaires were completed at baseline and after one year of follow up by 775 patients (response $67.5 \%$ ) and by 542 members of the general population (response $60.2 \%$ )

- Besides the eight scales of the SF36, a transition question on changes in health in the past year was included in the questionnaire

- Changes in all eight SF36 scales after a year were significantly related to changes in self reported health as measured by this question

- For patients with suspected peptic ulcer and varicose veins the SF36 profiles at one year were similar to the profiles of the general population

Box 7 Responsiveness of the SF36 health survey questionnaire to changes in health status ${ }^{13}$

and quality - for example, are certain groups of patients receiving substandard care or have substandard outcomes, do specific subgroups of care providers have excellent or inadequate performance, and which factors are decisive in acceptance and adoption of clinical guidelines by care providers?

Finally, research should be carried out on the advantages and disadvantages of internal (professionals, institutions, themselves) and external evaluations. What is the value of systems for recertification and reaccreditation of professionals, practices, and hospitals, and to what extent should self evaluation be the basis of such systems?

\section{Implementation of changes and improvement}

The next step in the quality improvement cycle is implementing new guidelines, procedures, and research findings and changing practice when necessary. Many care providers and institutions in health care have difficulties with changing routines, even when it is evident that improvements are required. Provision of care is often complex, and change usually demands both a positive attitude in different people and structural adaptations in the organisation. Research and development should particularly focus on the effectiveness, costs, and feasibility of different strategies for implementing new procedures and inducing change in normal clinical routines.

The results of systematic literature reviews disclose that continous medical education and dissemination of guidelines has a restricted effect, but systems for reminding, individual instruction, feedback by respected peers, support by experienced people, and the use of opinion leaders may be more effective. ${ }^{1516}$ Intensive strategies in particular, and a combination of interventions seem to be effective. ${ }^{16}$ It has, however, also become clear that the study designs in many controlled trials were inadequate; the interventions and the effect variables were not standardised.

Strategies that proved to be effective in one study were not effective in others. For instance, outreach visits in the study of Dittrich et $a l^{17}$ were effective in improving prevention of cancer; they were not effective in the study of
A randomised controlled trial measured the effects of consultations between GPs and orthopaedic surgeons on referral and interventions in the care for patients with locomotor problems

- For 18 months, 12 GPs held monthly joint consultation sessions with four participating orthopaedic surgeons

- Patients were seen by one surgeon in the presence of three GPs

- Patients were included in the trial when the GP was uncertain about the diagnosis or therapeutic management and if a referral was considered

- Patients were randomly assigned, by a blinded envelope procedure, to either the joint consultation sessions $(n=144)$ or a usual care control group ( $n=128)$

- A year later all the patients were examined by an independent orthopaedic surgeon

- There were significantly fewer referrals $(35 \% v$ $68 \%$ ) and diagnostic actions in the intervention group than in the control group

- More patients in the intervention group were symptom free at one year (35\% v 29\%)

Box 8 Effectiveness of joint consultations of GPs and surgeons: a randomised controlled trial ${ }^{14}$

Cockburn $e t a^{18}$ in stopping smoking. The outreach visitors in the second study spent on average 13 minutes in the practice, whereas those in the first study spent about six hours. Does this explain the difference in effect or was it the different proposals themselves, the nature of the health care setting, or other non-identified factors? Very little is known about precisely what elements work, or why: ${ }^{19}$

"cumulative progress in designing and refining successful interventions will be difficult to achieve until researchers begin looking inside the black box, measuring and analyzing the providers' beliefs, attitudes, reactions and judgements in some detail". ${ }^{19}$

Another problem is that research on many interesting strategies is still lacking. The effects are largely unknown of organisational development, team building, re-engeneering complex care processes with many care providers

The Society of Obstetricians and Gynaecologists in Canada in 1986 published guidelines on indications for performing caesarean sections

- To study which implementation strategy would have the largest impact on the actual application of the guidelines, a controlled study was set up among 76 doctors in 16 hospitals

- Hospitals and doctors were allocated to three groups:

One group received the guidelines by post One group received feedback on the results of a performance review

One group was actively approached by respected opinion leaders at work

- The results of before and after measurements in the three groups (chart audit) showed that the posted and the feedback groups did not perform differently, but the opinion leader group performed significantly differently

- In the opinion leader group there were $85 \%$ more vaginal births and $21 \%$ less caesarean sections; the duration of hospital stay was also considerably shorter

Box 9 Implementation of guidelines for performing caesarean section: testing the effectiveness of three methods ${ }^{21}$ 
involved, enhancing leadership in institutions, changing tasks and responsibilities of care providers, or introducing specific financial incentives and economic policies on causing change in practice performance. Particularly, features of the organisation may determine what happens in the "black box of quality improvement".

Little good research has been done on the costs, efforts, and time involved in the different strategies for changing practice. Methods to estimate the costs as well as the savings related to interventions need to be developed.

So, much research effort in the field of implementation of changes is required. The establishment of a Cochrane Centre on Effective Professional Practice (CCEPP), which coordinates reviews on methods for changing practice and implementing guidelines, will hopefully form an important stimulus for further research in this field. ${ }^{22}$

A systematic review of rigorous evaluations of the implementation of clinical guidelines that were published between 1976 and 1993, was performed after a search in DHSS-DATA, Embase, Medline, single databases, and bibliographies of related topics (only studies with a sound experimental design were included)

- Of 91 studies included, 81 reported significant improvements in the process of care after introducing guidelines

- In 12 out of 17 studies which measured patient outcomes, significant changes in outcomes were reported

- The conclusion from this systematic literature analysis was that:

Educational interventions requiring more active participation by professionals (targeted seminars, outreach visits, involvement of opinion leaders) are more likely to change performance Implementation strategies are more likely to be effective when they operate directly on the consultation between the professional and the patient (restructuring records, specific reminders during a consultation)

Box 10 Implementation of practice guidelines: a systematic literature review ${ }^{15}$

MANAGEMENT OF QUALITY IMPROVEMENT: SYSTEM AND CONDITIONS

A separate field of research on quality of care is concerned with good organisation and management of quality improvement. Quality improvement should be implemented through well designed programmes and should be supported by specific policies, structures, and resources.

Research in this area is scarce and may be focused on:

- Formal policies and regulations for quality improvement: what are the value and effect of specific laws on quality of care, systems for formal (re)licensing of professionals or (re)accreditation of institutions for health care, or of specific reward or payment systems related to implementation of quality improvement?

- Which organisational development is required for successful implementation of quality improvement; what is the role of quality managers, quality improvement teams, leadership, and team building?
To identify obstacles and requirements perceived by general practitioners in setting up quality improvement in their practices, in depth interviews were performed with a geographically stratified sample of $120 \mathrm{GPs}$ from single handed, paired, and group practices

- The interviews were performed by three experienced colleagues, who received a training of two half day sessions beforehand with feedback on some test interviews

- The interview consisted of a structured part (questionnaires to be completed by a GP) and a part with open questions

- The main problems with quality improvement mentioned by the GPs were:

Increase of workload in the practice Lack of time

No policies for quality improvement in the practice

Lack of agreement with colleagues about setting up quality improvement

- The perceived requirements for implementing it were:

Regular meetings with colleagues on quality improvement

Information and training on the aims, concepts and methods

Support on collecting data and audit in the practice and comparison with other practices Support on setting up peer review

Financial support for these activities

Box 11 Implementing quality improvement and audit in general practice: obstacles and requirements ${ }^{23}$

- What is the effect of specific programmes to teach care providers the concepts, skills, and methods of quality improvement?

- What time, staff, and money are needed for integrating quality improvement into normal work routines? Which support structures and tools for setting up quality improvement are desired (facilitators, computers, data bases, local audit committees)?

A randomised controlled trial with three groups of health professionals from health centres in Spain was set up

- Each goup contained 10 health centres One group received trainee centred training in quality improvement methods

One group received traditional training One group received no training

- Health professionals participating in the first group showed significantly higher scores on the appreciation of the training and on actual implementation of quality improvement activities than professionals in the other groups

Box 12 Effects of a problem based training to implement quality improvement activities among health professionals ${ }^{24}$

There are many questions in this area which urgently require an answer. Quantitative as well as qualitative research methodology (observation, interview) can be applied and the expertise from organisational research, healthcare policy making, and health economics is valuable.

\section{Setting up research on quality of care}

Research and development in quality of care aims at the scientific underpinning of theories, methods, tools, procedures, instruments, systems, programmes, policies, and conditions for assessing, improving, and managing the 
- GPs and county councils in Denmark agreed in 1995, that all 14 counties can spend 4.23 Dkr yearly per inhabitant over the age of 16 on continous medical education and quality improvement activities of general practitioners

- A total of 18 million Dkr (average $5700 \mathrm{Dkr}$ per GP) is available per year

- All counties will set up a quality improvement committee with representatives of GPs and politicians

- The task of the committee is the promotion of quality activities among GPs

- An evaluation is not yet planned

Box 13 Agreement on quality improvement between GPs and counties in Denmark

quality of patient care. It is clear that this type of research goes beyond the narrow interest of different separate disciplines and that expertise (theories, research methodology) from different disciplines and research fields is needed: for example from clinical epidemiology, sociology and psychology, psychometrics, medical informatics, quality of life research, economics, management sciences, and educational sciences.

Quantitative as well as qualitative methods from a range of different disciplines is required. Surveys, randomised controlled trials, psychometric analyses (reliability, validity, generalisability, responsiveness), programme evaluation, observations, case studies, and panel and group methods may all be used in this type of research.

Research and development in quality of care and quality improvement is strongly practice oriented. This causes some specific difficulties in designing and performing the research. It often proves to be difficult to set up well designed controlled trials or experiments in which confounding factors are under control and standardisation of the intervention is possible. The study often has to be linked and adapted to existing structures or wishes and demands of the study population (practices, professionals, institutions). It is often difficult to find the value of selected elements or factors in a quality improvement programme, as they are usually part of a broader, more comprehensive strategy. So, it is difficult to determine what is actually happening inside the "black box of quality improvement"; many interacting factors related to the study population and the study setting may play a part and may influence the ultimate effect and feasibility of a method or tool. A rigorous evaluation of the process is therefore usually important.

The preferable unit of analysis of the data is often not clear in research on quality of care. Is it really the patient? Or is it actually the professional, the unit, the practice, the hospital, or the whole region? Randomising patients or even professionals often cannot be assumed to be independent, and correction for correlation within clusters may be necessary.

An additional problem in research on quality of care is that it often has to use qualitative research methods. ${ }^{25}$ Such methods have not yet been fully developed in health care and are regarded as having limited value by many researchers in the field.

\section{Conclusions}

Quality of care as a new field of work has achieved a fixed and more or less stable position in health care. Care providers are increasingly faced with demands, through laws and regulations of health authorities and professional organisations, to set up systematic and continuous quality improvement at work. This is a new task for all healthcare professionals and institutions and needs effective and feasible tools, methods, instruments, and good theories. Good research is needed to find out how to manage this process and which methods are feasible and effective for it. We propose to view quality improvement as an activity related to practice, capturing a key position in between research on health and the patient on the one hand and research on health services on the other. Quality improvement translates the results of clinical trials and health technology assessment to daily practice, but also uses the results of research on factors in health services that influence the actual quality of care. ${ }^{3}$

"Health services need both research, that will develop new ideas and treatments and research that will help to evaluate them and ensure that those that are effective, are introduced rapidly into treatment". ${ }^{26}$

Many challenges can be identified for research on quality of care and quality improvement for the years to come. Priority should be given, for example, to the development of quality indicators, to evaluation of methods to develop clinical guidelines, to the study of methods to implement these guidelines successfully, to the research on methods to induce change in clinical practice, and to the research on management of quality improvement systems and processes. Not only the effectiveness of the various methods and tools should be studied, but also their efficiency: how to achieve a maximum effect with a minimum of costs and efforts. Particularly, research into the black box of improving practice and institutional performance should have high priority on the research agenda: which professional and organisational processes hinder or facilitate optimal care for patients? It is evident that such a research programme is a multidisciplinary undertaking. Not only quantitative research methods will be required, qualitative methods (interviews, observations, focus groups, etc) will also play an important part in the underpinning of the tools and instruments which will be used in future quality improvement systems. Separate research programmes with specific funding for research into quality of care are urgently required. Happily, a body of knowledge in this field is gradually being established. Part of this is by the publication of specific journals on quality of care and quality improvement with a research emphasis (Quality in Health Care, the International fournal on Quality in Health Care). Research on quality of care is an important field and has its own agenda. It must take its place alongside health services and clinical research. 
1 Grol R, Casparie A. Kwaliteit van zorg - onderzoek (quality of care research). Tijdschr Soc Gezondheidsz 1995;73:23744. (Abstract in English.)

2 Russel I, Wilson B. Audit: the third clinical science. Quality in Health Care 1992;1:51-5.

3 Field $M$, Tranquada R, Feasly J, eds. Health services research: work force and educational issues. Washington DC: National Academic Press, 1995.

4 Proctor S. Why clinical research needs medical audit. Quality in Health Care 1993;2:1-2.

5 Wensing $M$, Grol R, Van Montfort P, Smits A. Indicators of the quality of general practice care of patients with chronic illness: a step towards the real involvement of patients in the assessment of the quality of care. Quality in Health Care 1996;5:73-80.

6 McKee M. Routine data: a resource for clincal audit? Quality in Health Care 1993;2:104-11.

7 Kastein MR. Developing criteria for the evaluation of performance in family medicine using the delphi technique. Utrecht: University of Utrecht, 1994. (Thesis.)

8 Grimshaw J, Freemantle N, Wallace S, Russel I, Hurwitz B, Watt I, et al. Developing and implementing clinical practice guidelines. Quality in Health Care 1995;4:55-64.

9 Royal College of General Practitioners Clinical Guidelines Working Group. The development and implementation of clinical guidelines. London: RCGP, 1995.

10 Grol R, Thomas S, Roberts R. Development and implementation of guidelines for family practice: lessons from the Netherlands. 7 Fam Pract 1995;40:435-9.

11 Eccles M, Clapp Z, Grimshaw J, Adams P, Higgins B, Purves I, Russel I. Developing valid guidelines: methodological and procedural issues from the North of England Evidence Based Guideline Developing Project. Quality in Health Care 1996;5:44-50.

12 Baker R, Whitfield M. Measuring patient satisfaction: a test Baker $R$, Whitfield $M$. Measuring patient satisfaction: a test
of construct validity. Quality in Health Care 1995;1:104-9.

13 Garrat A, Ruta D, Abdalla M, Russel I. SF 36 health survey questionnaire II. Responsiveness to changes in health status in four common clinical conditions. Quality in Health Care 1994;3:186-92.

14 Vierhout W, Knottnerus J, Van Ooij A, Crebolder H, Pop P, Wesselingh-Megens A, Beusmans G. Effectiveness of joint consultation sessions of general practitioners and orthopaedic surgeons for locomotor system disorders. Lancet 1995;346:990-4

15 Grimshaw J, Freemantle N, Wallace S, Russel I, Hurwitz B, Watt I, et al. Developing and implementing clinical practice guidelines Quality in Health Care 1995;4:55-64

16 Wensing M, Grol R. Single and combined strategies for implementing changes in primary care: a literature review. Int $\mathcal{f}$ Qual Health Care 1994;6:115-32.

17 Diettrich A, O'Connor G, Keller A, Carney P, Levy D, Whaley F. Cancer: improving early detection and prevention. A community practice randomised trial. BMF 1992;304:687-91.

18 Cockburn J, Ruth D, Silagy C, Dobbin M, Reid Y, Scollo M, Naccarella L. Randomised trial of three approaches for marketing smoking cessation programmes to Australian marketing smoking cessation programmes
general practitioners. $B M \mathcal{F}$ 1992;304:691-4.

19 Kanouse D, Kallich J, Kahan J. Dissemination of effectiveness and outcomes research. Health Policy 1995;34:16792.

20 Robertson N, Baker R, Hearnshaw H. Changing the clinical behaviour of doctors: a psychological framework. Quality in Health Care 1996;5:51-4.

21 Lomas J, Enkin M, Anderson G, et al. Opinion leaders vs audit and feedback to implement practice guidelines. 7AMA 1991;265:2202-7.

22 Freemantle N, Grilli R, Grimshaw J, Oxman A. Implementing findings of medical research: the Cochrane Collaboration on Effective Professional Practice. Quality in Health Care 1995;4:45-7.

23 Grol R, Wensing M. Implementation of quality assurance and medical audit: general practioners' perceived obstacles and requirements. Br 7 Gen Pract 1995;45:548-52.

24 Saturno P. Training health professionals to implement quality improvement activities. Results of a randomised trial after one year of follow up. Int 7 Qual Health Care 1995;7:119-26.

25 Mays N, Pope C, eds. Quality research in health care. London: BMJ, 1996.

26 Smith R. The scientific basis of health services. BMF 1995; 311:961-2. 